TITLE:

\title{
Time- and Angle-Resolved Photoemission Spectroscopy of Hydrated Electrons Near a Liquid Water Surface
}

\section{AUTHOR(S):}

Yamamoto, Yo-ichi; Suzuki, Yoshi-Ichi; Tomasello, Gaia; Horio, Takuya; Karashima, Shutaro; Mitríc, Roland; Suzuki, Toshinori

\section{CITATION:}

Yamamoto, Yo-ichi ...[et al]. Time- and Angle-Resolved Photoemission Spectroscopy of Hydrated Electrons Near a Liquid Water Surface. Physical Review Letters 2014, 112(18): 187603.

ISSUE DATE:

2014-05-08

URL:

http://hdl.handle.net/2433/187024

RIGHT:

(C) 2014 American Physical Society 


\title{
Time- and Angle-Resolved Photoemission Spectroscopy of Hydrated Electrons Near a Liquid Water Surface
}

\author{
Yo-ichi Yamamoto, ${ }^{1}$ Yoshi-Ichi Suzuki, ${ }^{1,2}$ Gaia Tomasello, ${ }^{3}$ Takuya Horio, ${ }^{1,2,4}$ Shutaro Karashima, ${ }^{1}$ \\ Roland Mitríc, ${ }^{3}$ and Toshinori Suzuki ${ }^{1,2,4, *}$ \\ ${ }^{1}$ Department of Chemistry, Graduate School of Science, Kyoto University, Kitashirakawa-Oiwakecho, Sakyo-Ku, Kyoto 606-8502, Japan \\ ${ }^{2}$ RIKEN Center for Advanced Photonics, RIKEN, 2-1 Hirosawa, Wako 351-0198, Japan \\ ${ }^{3}$ Institut für Physikalishce und Theoretische Chemie, Universität Würzburg, Am Hubland, 97074 Würzburg, Germany \\ ${ }^{4}$ Japan Science and Technology Agency, CREST, Sanbancho, Chiyoda-ku, Tokyo 102-0075, Japan
}

(Received 27 January 2014; published 8 May 2014)

\begin{abstract}
We present time- and angle-resolved photoemission spectroscopy of trapped electrons near liquid surfaces. Photoemission from the ground state of a hydrated electron at $260 \mathrm{~nm}$ is found to be isotropic, while anisotropic photoemission is observed for the excited states of 1,4-diazabicyclo[2,2,2]octane and $\mathrm{I}^{-}$in aqueous solutions. Our results indicate that surface and subsurface species create hydrated electrons in the bulk side. No signature of a surface-bound electron has been observed.
\end{abstract}

DOI: 10.1103/PhysRevLett.112.187603

PACS numbers: 79.60.Cn, 73.20.-r, 73.40.-c, 82.80.Pv

Liquid water is indispensable for life, and its intriguing properties and dynamics due to strong hydrogen bonding attract great attention in wide areas in science and technology. The electronic structure of liquid water is approximated as a wide-gap semiconductor, in which an electron can be trapped in the forbidden band along with reorganization of hydrogen bonding around the electron [1]. The electron binding energy (EBE) of this trapped state (a hydrated electron) has been estimated as $3.4 \mathrm{eV}$ with respect to the vacuum level [2-6]. It has been shown that a low-energy electron undergoes dissociative attachment to biomolecules such as DNA bases [7], so that a hydrated electron and its excited states are also of interest in radiation chemistry and biology.

In addition to a hydrated electron in bulk water, it has been speculated that an electron is also trapped on the surface of water. In fact, a trapped electron on the surface of crystalline ice has been experimentally identified [8]. However, a surface-bound electron on liquid water is elusive [1]. A trapped state on the ice surface is longlived, because there is insufficient internal energy in ice to enable reorganization of a "frozen" hydrogen-bonding network and an electron cannot penetrate into the confined lattice of crystalline ice. On the other hand, liquid water has thermal fluctuations, which enables ultrafast response of the hydrogen-bonding network to an excess electron and, consequently, the electron rapidly penetrates into the bulk. Thus, a surface electron state of liquid water, if any, is expected to be short-lived; Madarász et al. have estimated the lifetime theoretically to be the 10 ps time scale [9].

Recently, Siefermann et al. claimed experimental observation of a surface-bound electron on liquid water with an electron binding energy (EBE) of $1.6 \mathrm{eV}$ and a lifetime at least as long as $100 \mathrm{ps}$ [2]. Their result, however, has not been confirmed by other experiments. Quantum chemical calculations by Uhlig et al. predict that an electron on water surface is rather well hydrated and has an EBE as large as $3.3 \mathrm{eV}$ [10], which is almost the same as the experimentally measured EBE value $(3.4 \mathrm{eV})$ of a hydrated electron in bulk water [2-6]. Theoretical treatments of a hydrated electron is still a subject of intense debates [11], and the accuracy of the theoretical EBE is unclear. Furthermore, EBE is a measure of the spatial size of an electron distribution but not of the location of the electron [12]. Thus, different experimental observables are necessary to explore a surfacebound electron.

In this Letter, we present femtosecond time- and angleresolved photoemission spectroscopy (TARPES) of hydrated electrons near a liquid surface. We create a hydrated electron by the charge transfer to solvent (CTTS) reaction from a photoexcited solute, because this scheme is more precisely controllable than multiphoton ionization of liquid water [2] and free from unwanted multiphoton-induced side reactions. Using surface-active solutes, charge transfer and generation of a hydrated electron primarily occur near the liquid surface, which is advantageous in searching for a surface-bound electron. We perform TARPES using a $260 \mathrm{~nm}(4.77 \mathrm{eV})$ probe pulse, because electronic inelastic scattering in water, prior to photoemission from the surface, is minimized when the kinetic energy is less than the band gap $(\sim 7 \mathrm{eV})$ of water [13-15]. The effective attenuation length (EAL) of an electron flux in water is estimated as $2-5 \mathrm{~nm}$ in our experiment [16-18]. On the other hand, photoemission anisotropy is reduced by elastic (and vibrationally inelastic) scattering in liquid water at low kinetic energies. Thus, the anisotropy may be observed only for the chemical species on the liquid surface.

The schematic diagram of our experiment is shown in Fig. 1. We discharge a liquid laminar flow from a fused silica capillary with a $25 \mu \mathrm{m}$ inner diameter into 


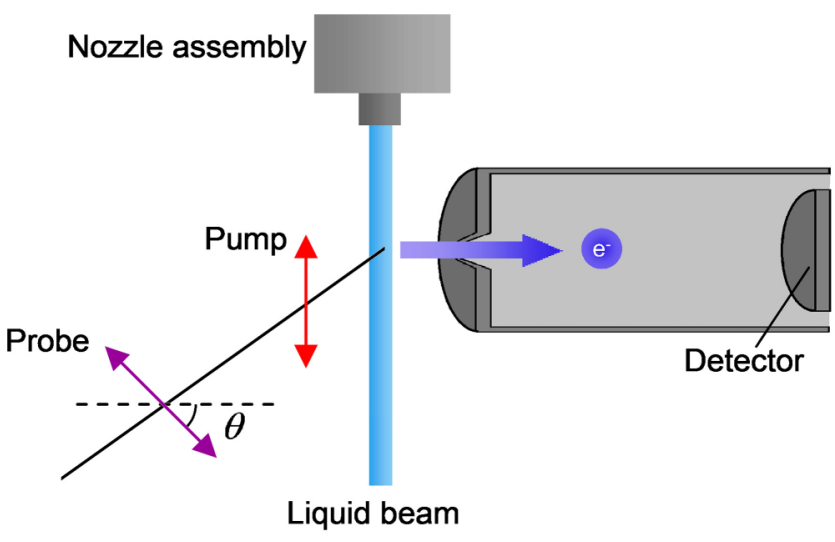

FIG. 1 (color online). Schematic diagram of our experimental appartus.

a time-of-flight (TOF) photoelectron spectrometer at a flow rate of $0.5 \mathrm{ml} / \mathrm{min}$. A femtosecond $226 \mathrm{~nm}$ pump pulse $(5.49 \mathrm{eV})$ electronically excites solutes in the liquid at $\sim 1 \mathrm{~mm}$ downstream from the nozzle, where the liquid temperature is estimated to be $275-280 \mathrm{~K}$. A femtosecond $260 \mathrm{~nm}$ probe pulse interrogates the nonstationary electronic state by photoemission. The cross correlation of the pump-probe pulses is $\sim 120$ fs in this study. Photoelectrons emitted from the liquid surface are sampled into the TOF analyzer through a $0.5 \mathrm{~mm} \phi$ skimmer located at $2 \mathrm{~mm}$ from the liquid surface. The electron flight path from the liquid surface to the detector is $1200 \mathrm{~mm}$, which provides an energy resolution of $\sim 50 \mathrm{meV}$. The $40 \mathrm{~mm}$ diameter of a microchannel plate detector provides a detection solid angle of $9 \times 10^{-4} \mathrm{sr}$. A high-repetition-rate $100 \mathrm{kHz}$ laser system is employed to compensate for the small signal due to the small detection solid angle [19]. The linear polarization of the pump pulse is fixed perpendicular to the electron detection axis, and angle-resolved photoemission spectra are measured by rotating the linear polarization of the probe pulse from 0 to $90^{\circ}$ with respect to the electron detection axis. The time profile of the signal from the microchannel plate is recorded using a multichannel scaler. When angular resolution is not required, the spectrometer is also used as a magnetic bottle photoelectron spectrometer [20].

Let us first examine aqueous DABCO (1,4-diazabicyclo $[2,2,2]$ octane) solution. The DABCO molecule has three aliphatic $\left(-\mathrm{CH}_{2}-\mathrm{CH}_{2}-\right)$ bridges, which make this molecule hydrophobic and segregated on the surface. In order to ascertain its enhanced concentration on the surface, we performed soft-x-ray photoemission spectroscopy of aqueous DABCO solution at the BL17SU beam line at the synchrotron radiation facility, SPring-8. We compared $\mathrm{N}(1 s)$ photoemission from aqueous $0.25 \mathrm{M}$ DABCO solution and aqueous $0.25 \mathrm{M}\left(\mathrm{NH}_{4}\right)_{2} \mathrm{SO}_{2}$ solution, because $\mathrm{NH}_{4}^{+}$is not surface active [21]. The observed $\mathrm{N}(1 s)$ photoelectron intensity (binding energy of $\sim 400 \mathrm{eV}$ ) of aqueous DABCO solution is 5 times stronger than aqueous
$\left(\mathrm{NH}_{4}\right)_{2} \mathrm{SO}_{2}$ solution at the photon energy of $440 \mathrm{eV}$. Thus, we confirmed that DABCO has an enhanced molecular density on the liquid surface.

The low-lying excited states of DABCO in the gas phase are Rydberg states for which strong photoemission anisotropy has been observed [22]. The UV absorption spectrum of aqueous DABCO solution exhibits a gradually increasing intensity from $240 \mathrm{~nm}$. The theoretical absorption spectrum is presented in the Supplemental Material [23], as Fig. S1, along with the assignments of the electronic transitions. The four lowest excited states of DABCO in water have dominant $s$ and $p$ Rydberg characters in agreement with those states in the gas phase, although the Rydberg orbitals in water have delocalization over unoccupied orbitals of water molecules. We performed TARPES of aqueous $0.5 \mathrm{M}$ DABCO solution using a $226 \mathrm{~nm}$ pump pulse and a $260 \mathrm{~nm}$ probe pulse. Figure 2(a) presents the time-energy two-dimensional map of the observed photoelectron spectra: EBE is the difference between the probe photon energy $(4.77 \mathrm{eV})$ and the observed photoelectron kinetic energy. The observed EBE has been calibrated for the streaming potential of a liquid beam. This map was determined using a magnetic bottle TOF method, so that the signal was integrated over the photoemission angle. Figure 2(a) clearly shows that the EBE rapidly increases within 1 ps and reaches $3.4 \mathrm{eV}$, which is the EBE of a hydrated electron. The result clearly reveals ultrafast CTTS from the excited state of DABCO to liquid water, which is also supported by our nonadiabatic quantum mechanics-molecular mechanics (QM-MM) simulation [23]. As seen in Figs. S2 and S3 in the

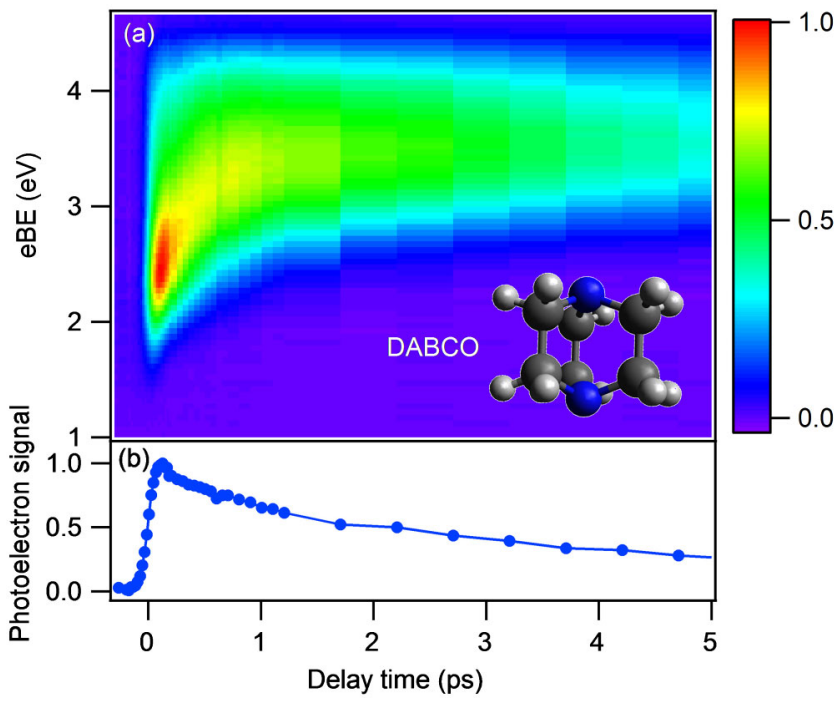

FIG. 2 (color online). (a) Two-dimensional false color map of photoelectron spectra measured for aqueous $0.1 M$ DABCO solutions at different pump-probe time delays. The pump and probe laser wavelengths are 226 and $260 \mathrm{~nm}$, respectively. (b) The total electron intensity profile obtained from (a) by integrating the distribution at each delay time over EBE. 
Supplemental Material [23], the simulation predicts that initial photoexcitation is followed by internal conversion down to the lowest excited state of DABCO. The $S_{1}$ state has a rather large Mulliken charge on the water molecules, while the electron is still strongly interacting with the DABCO cation before significant reorganization of the hydration structure enables diffusional separation of the ion and the electron. Figure 2(b) shows the total photoelectron intensity profile obtained from Fig. 2(a) by integrating the distribution at each delay time over EBE.

Figure 3 presents TARPE spectra measured for the same CTTS reaction from DABCO to water, except that the concentration has been increased to $0.5 \mathrm{M}$ to compensate for low signal intensities in angle-resolved measurements. Each upper panel shows a photoelectron spectrum, $S(E, \theta)$, at different time delays from 100 fs to 3 ps, respectively. Rapid change of the photoelectron spectrum appears predominantly in the low EBE region, where strong photoemission anisotropy is observed. The intensity is minimized when the probe laser polarization is perpendicular to the electron detection axis. The photoemission signal, corresponding to EBEs of $1.7-2.7 \mathrm{eV}$ at $100 \mathrm{fs}$, is characterized by an anisotropy parameter $\beta$ of $0.3 \pm 0.1$ defined by the following equation:

$$
I(\theta)=1+\frac{\beta}{2}\left(3 \cos ^{2} \theta-1\right),
$$

where $\theta$ is the angle between the probe laser polarization and the electron detection axis (a higher term has been neglected). The observed anisotropy is considerably smaller than the value observed for DABCO in the gas phase [22]. The reduction of the anisotropy parameter is most likely caused by electron scattering by a hydration shell. To examine this possibility theoretically, we have simulated

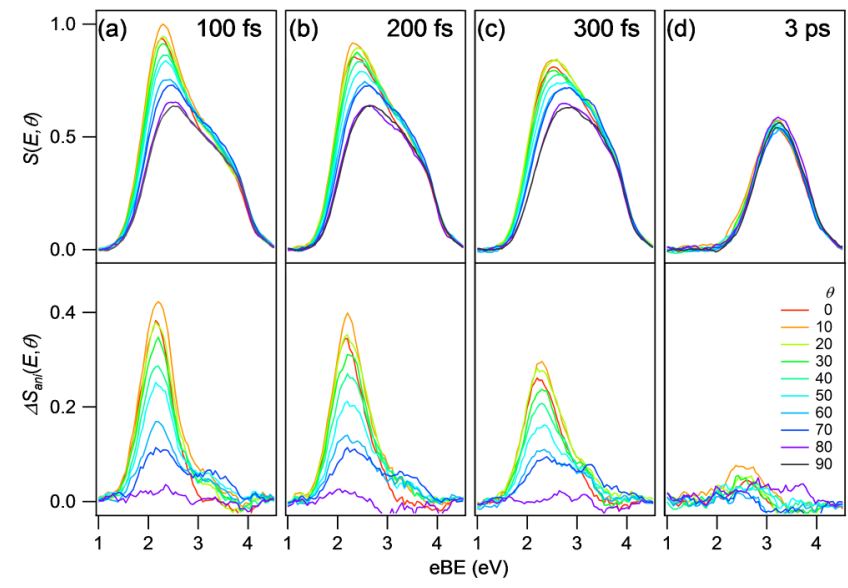

FIG. 3 (color online). Photoelectron spectra of aqueous $0.5 \mathrm{M}$ $\mathrm{DABCO}$ solution as a function of the polarization angle of the probe pulse $\theta$ with respect to the electron detection axis. The spectra were observed at different pump-probe time delays of (a) $100 \mathrm{fs}$, (b) $200 \mathrm{fs}$, (c) $300 \mathrm{fs}$, and (d) $3 \mathrm{ps}$. the photoemission anisotropy of hydrated DABCO using continuum multiple scattering $X \alpha$ calculations[23]. We assumed the $3 s$ Rydberg state of DABCO hydrated by 64 water molecules. The calculated photoemission distribution was isotropic. Thus, weak yet finite photoemission anisotropy observed for aqueous DABCO solution is ascribed to DABCO molecules segregated on the liquid surface.

For closer examination of the anisotropic photoemission component, we calculated the difference spectrum defined by $\Delta S_{\text {ani }}(E, \theta)=S(E, \theta)-S\left(E, 90^{\circ}\right)$ at each time delay, as shown in the lower panels of Fig. 3. $\Delta S_{\text {ani }}(E, \theta)$ at $100 \mathrm{fs}$ exhibits a Gaussian band shape centered at $2.2 \mathrm{eV}$. As time goes from 100 to $300 \mathrm{fs}, \Delta S_{\text {ani }}$ gradually diminishes and no anisotropic component is discernible at 3 ps. The EBE distribution with a peak at $3.3 \mathrm{eV}$, seen in Fig. 3(d), is most likely of a hydrated electron, although it is still under electrostatic interaction with $\mathrm{DABCO}^{+}$at this short time delay. Figure 3(d) also indicates that photoemission from a hydrated electron is isotropic. The photoemission at a longer delay time of $10 \mathrm{ps}$ was essentially the same. Thus, isotropic photoemission of the hydrated electron produced from DABCO indicates that the electron is not at the liquid surface

In order to further elucidate the nature of the hydrated electron produced by CTTS in the near surface region, we next consider an atomic anion as a solute. Although atomic ions have been believed to be repelled from the liquid surface by image forces, recent theoretical and experimental studies have shown that ions with large polarizabilites have enhanced densities on a water surface [24,25]. The surface concentration of anions is enhanced by counter cations. For example, tetrabutyl ammonium ion $\left(\mathrm{TBA}^{+}\right)$is hydrophobic and segregated on the liquid water, and $\mathrm{TBA}^{+}$ drags $\mathrm{I}^{-}$to the surface for charge neutrality requirement [26-28]. Consequently, $\mathrm{I}^{-}$in an aqueous $25 \mathrm{mM}$ TBAI solution is estimated to be 70 times more concentrated in the subsurface region than the bulk [29].

Figures 4(a) and 4(b) show TARPE spectra of aqueous $50 \mathrm{mM}$ TBAI solution measured at $100 \mathrm{fs}$ and $100 \mathrm{ps}$, respectively. A $226 \mathrm{~nm}$ pump pulse excites hydrated $\mathrm{I}^{-}$to a metastable electronic state, which undergoes CTTS to create a hydrated electron and a neutral I atom [30]. The $S(E, \theta)$ at 100 fs is clearly asymmetric, similar to the case of DABCO. The $\beta$ value in the EBE range of $1.3-2.3 \mathrm{eV}$ is $0.15 \pm 0.1$. The anisotropic component $\Delta S_{\text {ani }}(E, \theta)$ has its band center at $1.8 \mathrm{eV}$. On the other hand, at $100 \mathrm{ps}$ [Fig. 4(b)], $S(E, \theta)$ exhibits a symmetric band shape centered at $2.8 \mathrm{eV}$. This EBE value is clearly smaller than the $\operatorname{EBE}(3.4 \mathrm{eV})$ of a hydrated electron in bulk water [6]. Since we have calibrated the observed EBE to account for a streaming potential, the difference is not ascribed to charging of the liquid beam. Previous soft-x-ray photoemission spectroscopy has suggested that a segregated monolayer of TBAI is created at a concentration of $25 \mathrm{mM}$ [29]; the 


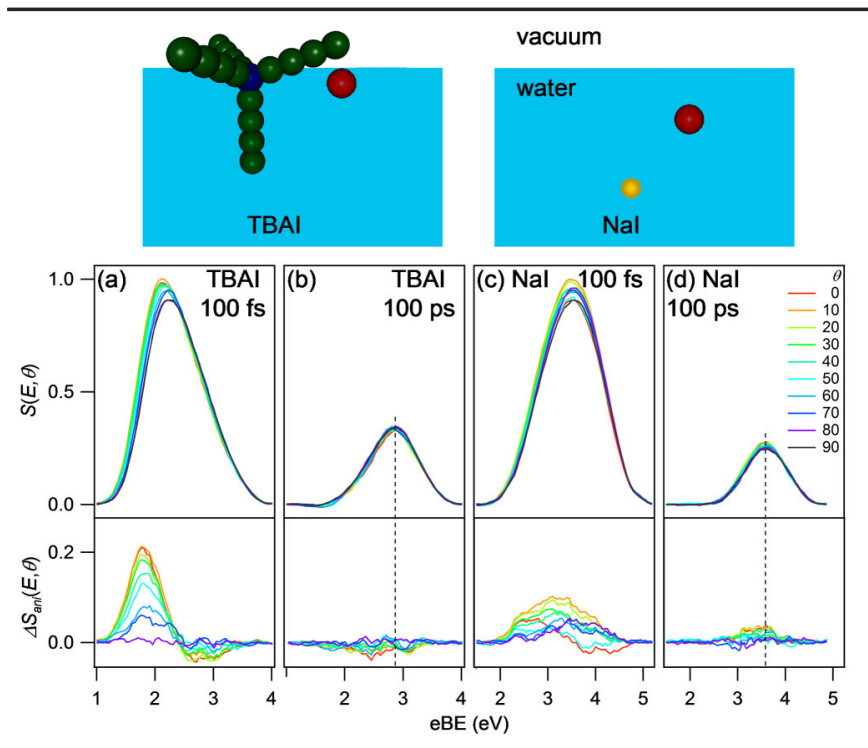

FIG. 4 (color online). Photoelectron spectra of aqueous $50 \mathrm{mM}$ TBAI solution [(a) and (b)] and $0.5 M \mathrm{NaI}$ solution [(c) and (d)] as a function of the polarization angle of the probe pulse $\theta$ with respect to the electron detection axis. The spectra were observed at different pump-probe time delays of $100 \mathrm{fs}$ [(a) and (c)] and 100 ps [(b) and (d)].

concentration we employed in this study is two times higher than this value. The small EBEs indicate the hydrated electron is created in the subsurface region, under the influence of electrostatic interaction with the $\mathrm{TBA}^{+}$layer. The full account of this trapped electron in the segregated layer is reported elsewhere.

Figures 4(c) and 4(d) present TARPE spectra measured for aqueous $0.5 \mathrm{M} \mathrm{NaI}$ solution at $100 \mathrm{fs}$ and $100 \mathrm{ps}$, respectively. The main difference between the cases of TBAI and NaI is their energetics. As mentioned earlier, the electron binding energies of the transient electronic states and a solvated electron are clearly smaller in aqueous TBAI solution than in NaI solution. The electron binding energy of a hydrated electron in aqueous $\mathrm{NaI}$ solution is in agreement with the values measured using other solutions [2-6]. The photoemission anisotropy, if any, is very small; the $\beta$ value determined for EBEs of $3.0-4.0 \mathrm{eV}$ is 0.03 at $100 \mathrm{fs}$, which is in qualitative agreement with a previous one-color two-photon photoemission experiment by Tang et al. [13]. The $0.5 \mathrm{M}$ aqueous NaI solution (10 times more concentrated than the aqueous TBAI solution) has a sufficient $\mathrm{I}^{-}$density in the subsurface region to exhibit weak photoemission anisotropy. However, the EBE of the anisotropic component is the same as that of isotropic component, suggesting that $\mathrm{I}^{-}$in the subsurface region of the aqueous NaI solution is well hydrated, and that the CTTS reaction produces a hydrated electron in the bulk. We performed continuum multiple scattering $X \alpha$ calculations [23] using snapshot geometries of a hydrated electron in bulk water calculated by Uhlig et al. [31]. For simplicity, we considered 64 and 125 water molecules around the center of mass of the excess electron. The calculated photoemission anisotropy parameters for the 125 water molecules were $0<\beta<0.5$ and smaller than that for the 64 water molecules in the photoelectron kinetic energy range of $0-6 \mathrm{eV}$, in qualitative agreement with the experimental result.

In conclusion, our first TARPES of aqueous solutions revealed that anisotropic photoemission occurs from transient electronic states of species segregated on liquid surfaces. These short-lived states, exhibiting smaller EBEs than a hydrated electron in bulk water, decay in 1 ps. Within this short time range, an electron is still strongly interacting with the precursor and not regarded as a hydrated electron. We identified no signature of the previously suggested band of a surface-bound electron at an $\mathrm{EBE}$ of $1.6 \mathrm{eV}$ with a long lifetime [2]. Isotropic photoemission was observed for a hydrated electron even if it was produced from $\mathrm{DABCO}$ on the surface, indicating that the CTTS reaction from the surface species creates a hydrated electron in the bulk side of liquid. The observed isotropic photoemission from a hydrated electron is not inconsistent with a cavity state of a hydrated electron, because even photoemission from an electronic state with a strong $s$ character can be isotropic owing to scattering by the hydration shell.

We thank N. Kurahashi for experimental assistance in soft-x-ray photoemission spectroscopy and P. Jungwirth and F. Uhlig for providing us with snapshot geometries of a hydrated electron. Numerical calculations were conducted on the RIKEN Integrated Cluster of Clusters. The synchrotron radiation experiments were performed at BL17SU of SPring-8 with the approval of RIKEN (Proposal No. 20130075).

*suzuki@kuchem.kyoto-u.ac.jp

[1] L. Turi and P. J. Rossky, Chem. Rev. 112, 5641 (2012).

[2] K. R. Siefermann, Y. Liu, E. Lugovoy, O. Link, M. Faubel, U. Buck, B. Winter, and B. Abel, Nat. Chem. 2, 274 (2010).

[3] Y. Tang, H. Shen, K. Sekiguchi, N. Kurahashi, T. Mizuno, Y.-I. Suzuki, and T. Suzuki, Phys. Chem. Chem. Phys. 12, 3653 (2010).

[4] A. Lübcke, F. Buchner, N. Heine, I. V. Hertel, and T. Schultz, Phys. Chem. Chem. Phys. 12, 14629 (2010).

[5] A. T. Shreve, T. A. Yen, and D. M. Neumark, Chem. Phys. Lett. 493, 216 (2010).

[6] T. Horio, H. Shen, S. Adachi, and T. Suzuki, Chem. Phys. Lett. 535, 12 (2012).

[7] L. Sanche, Eur. Phys. J. D 35, 367 (2005).

[8] J. Stähler, M. Mehlhorn, U. Bovensiepen, M. Meyer, D. O. Kusmierek, K. Morgenstern, and M. Wolf, Phys. Rev. Lett. 98, 206105 (2007).

[9] A. Madarász, P. J. Rossky, and L. Turi, J. Chem. Phys. 126, 234707 (2007)

[10] F. Uhlig, O. Marsalek, and P. Jungwirth, J. Phys. Chem. Lett. 4, 338 (2013). 
[11] R. E. Larsen, W. J. Glover, and B. J. Schwartz, Science 329, 65 (2010).

[12] O. Marsalek, F. Uhlig, T. Frigato, B. Schmidt, and P. Jungwirth, Phys. Rev. Lett. 105, 043002 (2010).

[13] Y. Tang, Y.-I. Suzuki, H. Shen, K. Sekiguchi, N. Kurahashi, K. Nishizawa, P. Zuo, and T. Suzuki, Chem. Phys. Lett. 494, 111 (2010).

[14] M. Michaud, A. Wen, and L. Sanche, Radiat. Res. 159, 3 (2003).

[15] T. Suzuki, Int. Rev. Phys. Chem. 31, 265 (2012).

[16] F. Buchner, T. Schultz, and A. Lübcke, Phys. Chem. Chem. Phys. 14, 5837 (2012).

[17] S. Thürmer, R. Seidel, M. Faubel, W. Eberhardt, J. C. Hemminger, S. E. Bradforth, and B. Winter, Phys. Rev. Lett. 111, 173005 (2013).

[18] K. Nishizawa, Y.-I. Suzuki, T. Suzuki, and N. Kurahashi (to be published).

[19] H. Shen, S. Adachi, T. Horio, and T. Suzuki, Opt. Express 19, 22637 (2011).

[20] P. Kruit and F. H. Read, J. Phys. E 16, 313 (1983).

[21] S. Gopalakrishnan, P. Jungwirth, D. J. Tobias, and H. C. Allen, J. Phys. Chem. B 109, 8861 (2005).

[22] L. Poisson, R. Maksimenska, B. Soep, J.-M. Mestdagh, D. H. Parker, M. Nsangou, and M. Hochlaf, J. Phys. Chem. A 114, 3313 (2010).
[23] See Supplemental Material at http://link.aps.org/supplemental/ 10.1103/PhysRevLett.112.187603 for the details of QM-MM simulation of the solvated DABCO and continuum multiple scattering $X \alpha$ calculations for hydrated DABCO and an electron.

[24] P. Jungwirth and D. J. Tobias, Chem. Rev. 106, 1259 (2006).

[25] S. Ghosal, J. C. Hemminger, H. Bluhm, B. S. Mun, E. L. D. Hebenstreit, G. Ketteler, D. F. Ogletree, F. G. Requejo, and M. Salmeron, Science 307, 563 (2005).

[26] F. Eschen, M. Heyerhoff, H. Morgner, and J. Vogt, J. Phys. Condens. Matter 7, 1961 (1995).

[27] C. Wang and H. Morgner, Appl. Surf. Sci. 257, 2291 (2011).

[28] S. Holmberg, R. Moberg, Z. C. Yuan, and H. Siegbahn, J. Electron Spectrosc. Relat. Phenom. 41, 337 (1986).

[29] B. Winter, R. Weber, P. M. Schmidt, I. V. Hertel, M. Faubel, L. Vrbka, and P. Jungwirth, J. Phys. Chem. B 108, 14558 (2004).

[30] Y.-I. Suzuki, H. Shen, Y. Tang, N. Kurahashi, K. Sekiguchi, T. Mizuno, and T. Suzuki, Chem. Sci. 2, 1094 (2011).

[31] F. Uhlig, O. Marsalek, and P. Jungwirth, J. Phys. Chem. Lett. 3, 3071 (2012). 